\title{
AN INTERACTIVE CODE SUPERLANS FOR EVALUATION OF RF-CAVITIES AND ACCELERATION STRUCTURES
}

\author{
Myakishev D.G., Yakovlev V.P. \\ Institute of Nuclear Physics, Novosibirsk, 630090, USSR
}

\section{Abstract}

The new code SUPERLANS is described. The main SUPERLANS ieatures are: an evaluation of both the standing waves in axisymmetrical cavities and travelling waves in periodic structures; the number of modes (or dispersion curves) are calculated simultaneously in the arbitrary spectra domain. To increase the code efficiency the following modern methods are used: the isoparametric second order finite elentents for the field approximation; the subspace iteration method for the determination of spectra. To increase the aceuracy of calculation of the field near the axis the coordinates $\left(Z, R^{2}\right)$ are used. There is code version for IBM PC/AT with the inleractive dialogue data input and postprocessor with developed graphics. The code is currently used for the INP RF-system design.

\section{INTRODUCTION}

In 1979 the LANS [1] code was developed in the INP. The code used the inverse iteration methor with a spectra shift for the calculation of the eigen frequencies in the arbitrary spectra domain in axisymmetrical RF-cavitics. The triangular simplex elements were used in the code. Then other codes MLLTIMODE [2] and PRLD [3] were developed. The subspace iteration method is used for the simultaneous calculation of the number of modes in the codes. The codes also permits to calculate the dispersion curves in periodic structures. The isoparametric second order elements are used in the codes. We develop new code SI:I'ERLANS based on the me thods which are used in these codes. The main leatures of new code are:

- the code is made for personal computer and has interactive input-output and uses the graphical possibilities of the computer:

the problem formulation is used, which eliminates the singularity in! matrix elements on axis $[2,3]$;

- the coordinates $\left(Z, R^{2}\right)$ are used for the increase in accuracy of field calculations near axis.

\section{GENERAL}

The code SUPERLANS was made for the calculation of azimuthally-homogeneous modes in the axisymmetrical RF cavities and periodic structures. For the evaluation of eigen modes the wave equation with respect to following function is solved: function:

$$
H=\left\{\begin{array}{l}
r \cdot H_{0}-f: \text { wave } \\
r \cdot E_{i}-H \text { wave }
\end{array}\right.
$$

The boundary conditions are set on axis, plane of symmctry and metallic surfaces. The metallic surfaces are supposed to have an ideal conductivity. The grid with eight node isoparametric elements is used in the code. The Galerkin method is used to obtain an algebraic system $\left(\mathrm{A}-k^{2} \cdot \mathrm{B}\right) \cdot[\mathrm{I}=0$. Unlike $[2,3]$ the integration in the Galerkin method is made over the volume problem, but not over the square of the cavity crossection. In this case, the matrix coefficients haven't the singularity in integral expression:

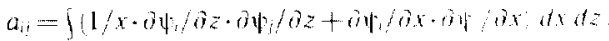

$$
\begin{aligned}
& b_{i j}=w_{1} \cdot w_{i} / x d x d z, \text { where } x=r^{2}+4
\end{aligned}
$$

The Dirichlet boundary condition is set on axis, so the matrix coefficients aren't calculated for the axis nodes. The basic functions and their derivatives with respect to $z$ for the nodes near axis are proportional to $x$, so the mat rix coefficients haven't singularity in the integral expression on axis.

The reason of using the coordinate system $\left(Z, R^{2} / 4\right)$ is the following. The function $I 1$ is proportional to $r^{2}$ near axis, since the field is proportional to $r$ near axis. lsoparametric elements are closed to rectangle near axis, so, if the ordinary coordinate system is used, the $r$ and $z$ coco dinates are proportional to the corresponding local coordi nates. As a result, the function 11 and $r$ hehave similarly near axis, so 11 is proportional to $r$. Therelore, the coordinate system $\left(Z, R^{2} / 4\right)$ is put into operation to remove this uncorrectness in the iield deseription. In this case, we must to move the middle nodes on elements near axis for the right description of coordinates on element. The node on element side perpendiculas to the axis is moved. The radius of node is found from the cyuation $r^{2}=r_{1} / 2$, where $r_{n}$ - the radius of corner node

As we have said above, SUPERLANS permits to cal. culate the travelling waves in periodic stmotures. The calculation of the dispersion curves is made automatically. The Floquet theorem [4] is used ior the calculation of travelling waves. The theorm permits to build the travelling wave with the phase shift 0 over the structure period by two real functions. One of them is symmetrical on the period, the other is antisymmetrical. The efuations for this functions are analogous to the equation bor the standing wave. The iunctions are related on hound of period by coefficients dependent of the phase shift $\theta$.

The code also permits the evaluation of the RF cavily with an inhomogeneous ferromagnetic and dielectric iilling. For example, the one version of cavity with the retu. ning of frequency for the LEB [5] is shown below

The code is realized on IBM PC/AT and the graphic possibilities of computer are widely used. The interactive input-output are used with simultaneous displaying graphical information. This possibility simplifies the procedure of cavity geometry input. The logical mesh with maximum number of nodes 3000 is used for generating the finite element mesh. Ten modes can be itcrating simultaneously in the arbitrary spectra domain.

The code applications are shown on some examples.

The field distributions of two modes in DAW structure [6] are shown in Figs. $1 a$ and $1 b$, and dispersion curve is shown in Fig. $1 c$.

The calculation of RF cavity of VEPP-5 $[7]$ is given in the Figs. $2 a, 2 b$. The wide spectrat dumain was explored (200 modes) for this cavity. The working and one of the highest quasi-optic modes are shown in figures.

The results of cavity calculation for accelerator « $\mathrm{Si}$ 
beria» [8] are given in Figs. $3 a, 3 b$. In this case the modes, interacting with a beam, are shown.

The two iirst modes of cavity of accelerator «lLU-10» [9] are shown in Figs. 4a, 4b. The specific feature of this cavity is that it consists of two parts.

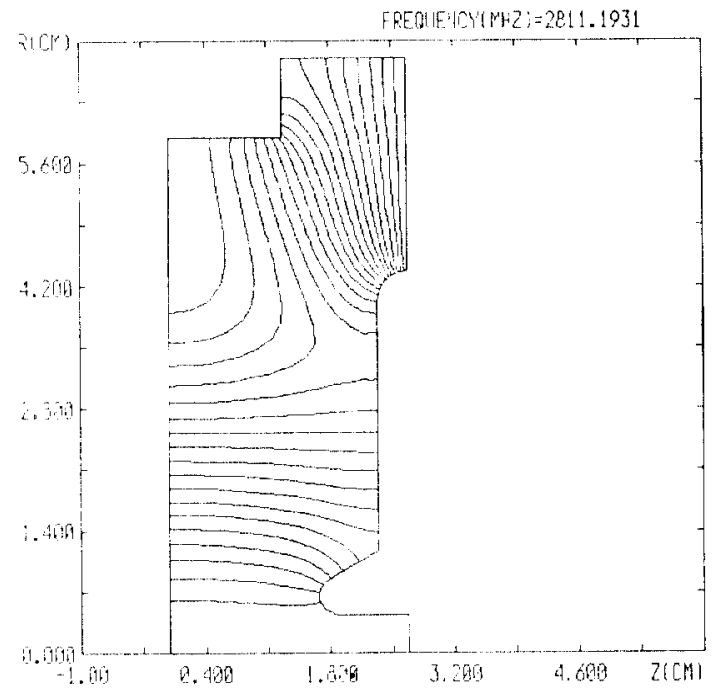

Fig. $1, a$
The constant voltage is applied to the internal part to suppress the multipactor.

The calculation result of the one version of cavity for the LEB with an inhomogeneous ierromagnetic and dielectric filling is shown in Fig. 5. This cavity is tuned in $\mathrm{RF}$ frequency within $30 \%$.

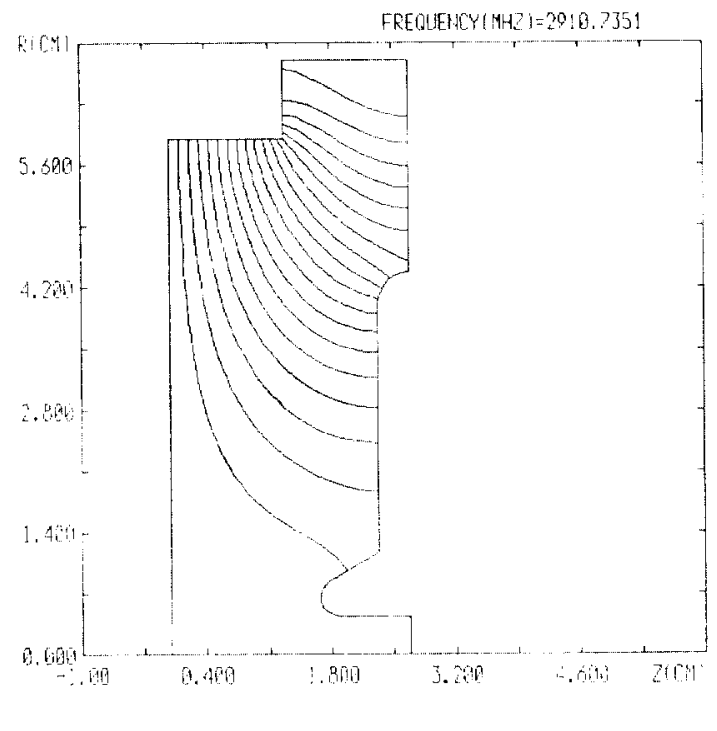

Fig. $1 . b$

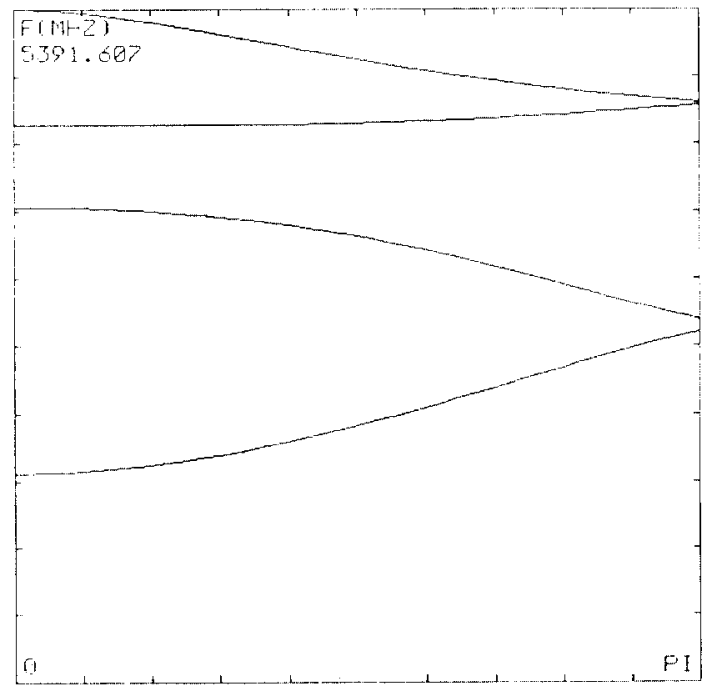

Fig. $1, c$

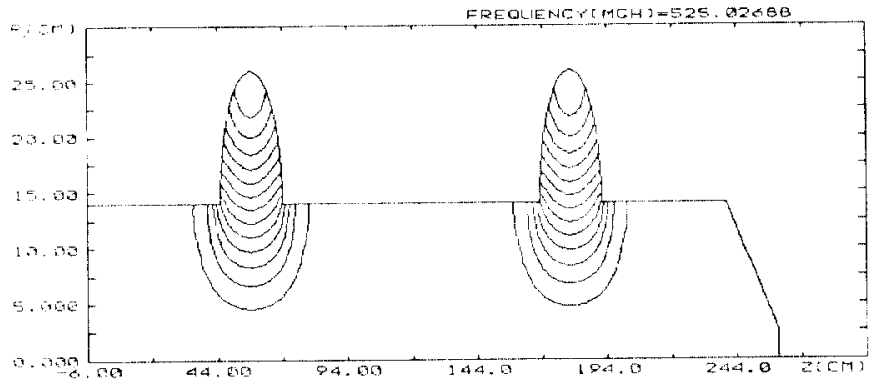

Fig. 2,a

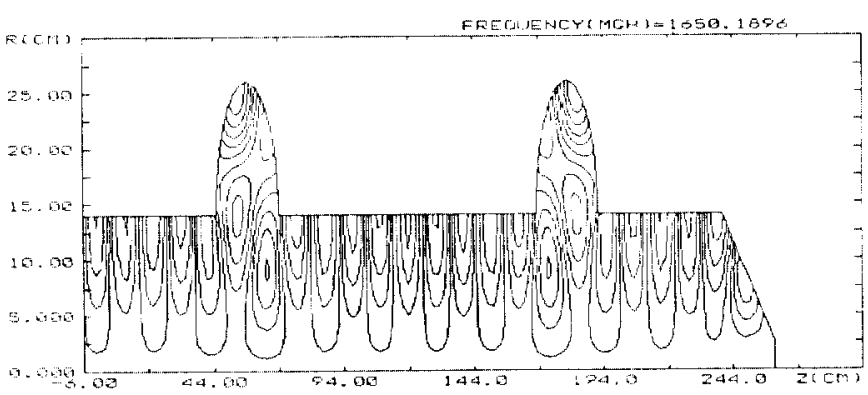

Fig. 2,h 


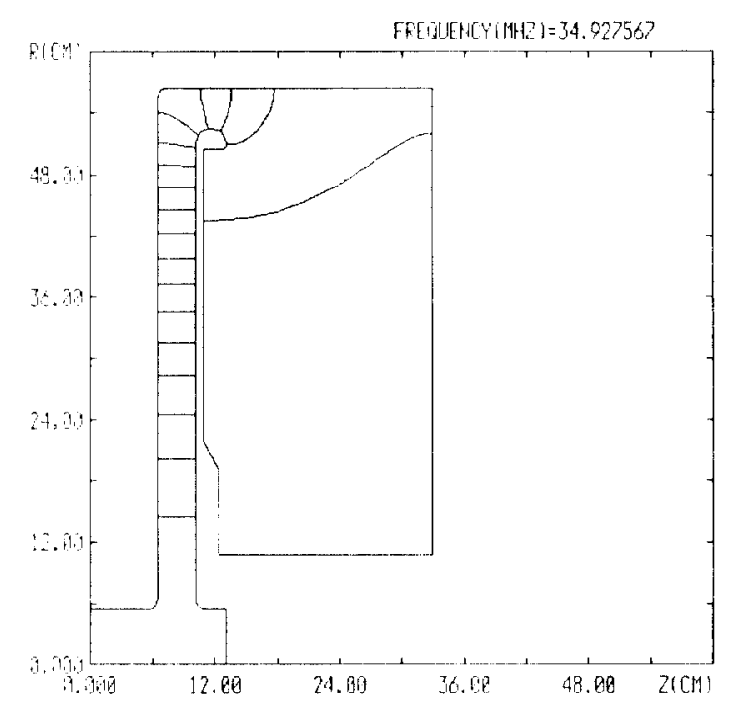

Fig. 3,a

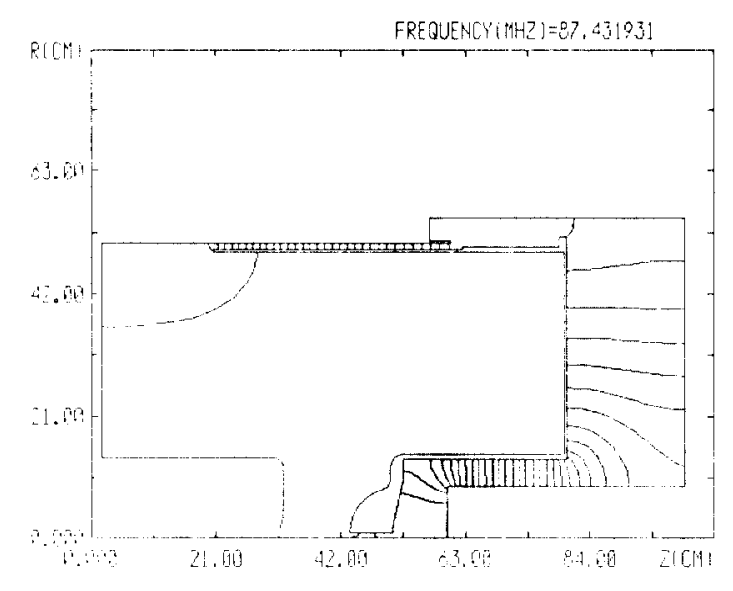

Fig. $4 . a$

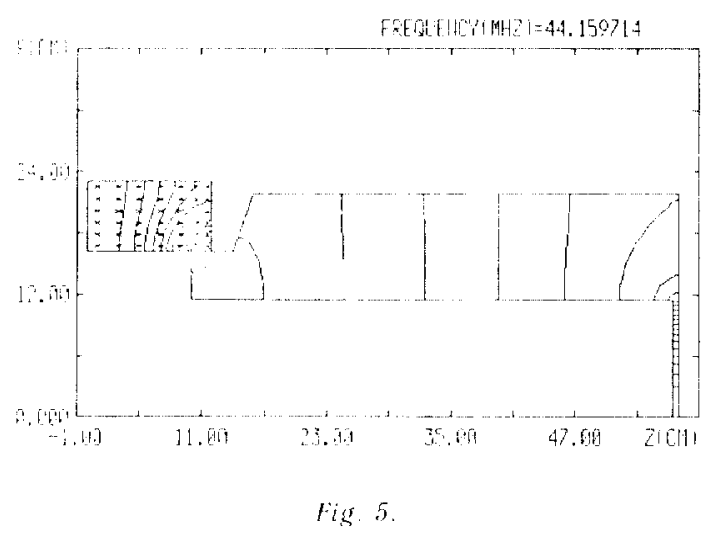

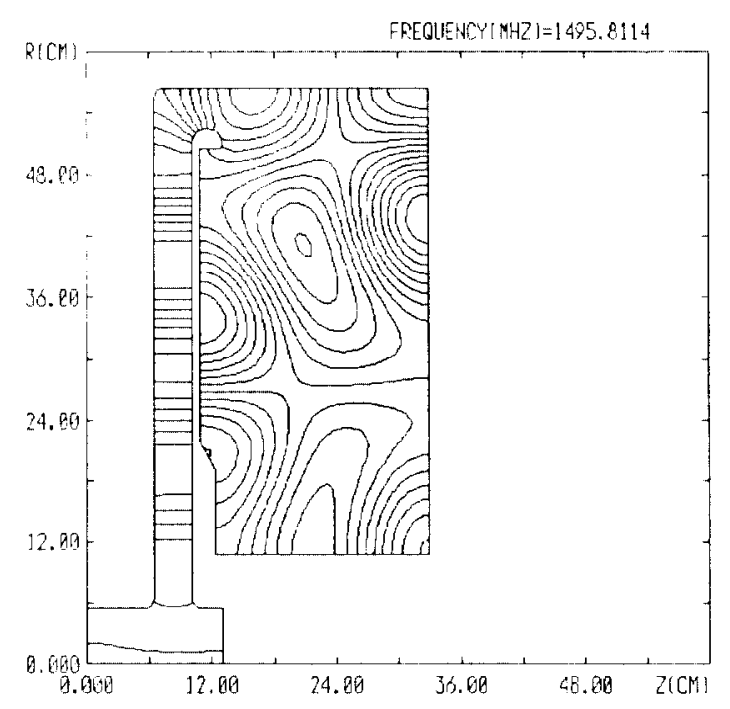

Fig. $3, b$

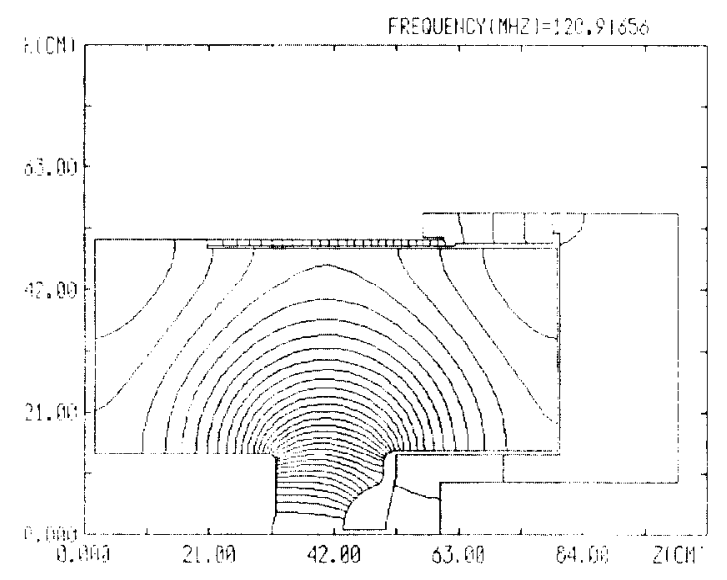

Fig. 4.6

\section{REFERENCES}

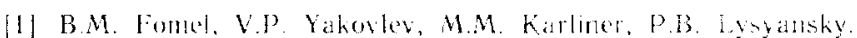
LANS - A Now Conde for Evaluation of the Electromagnetic Fields and Resonance Frecpuefoles of Axisymmetrian RF Cavities. Particle Accelerators, N 3, 1981, vol.11, pp $173 \ldots .179$.

II MS. Kaschiev et al Procedings of the 9th All Lnion Conie rence on Charged Particle Accelerators. Dubna, USSR, 16 Oet. 1984, INIS-SU-352, CONF-8410203, Vol.1, pp.137-141.

[3] A.G. Disikovety at al. "PRUD-W: A New Code to Compute and Design Acculerating Struturess, Particle Accelerators N 1, 1986, vo! 20, pp.23 43

44 Bell M. Donle G. CIERN $73-1$, Geneva, 1973

15) Site-Specific Conceptual Design, SSCL-SR-1056, July 1990.

[6] M.M. Karliner et al. Proc of the EPAC. Rome, June 7-11. 1988, vol.1, pp.602-604.

17] V.A Lebeder et al Proc of the 12 tin All-Union Conference on Charged Particle Accelerators, Diblla, USSR, 3-5 Oct.1990, to be published.

|8| V.V. Anashin et al. Proc oi the FPAC. Rome, June 7--11, 1988. vol.1. p.57.

(9) V.I. Auslender et al. To be Published 